\title{
Global ChaOs SynCHRONIZATION OF SPROTT-L AND SPROTT-M SYSTEMS BY ACTIVE CONTROL
}

\author{
Sundarapandian Vaidyanathan \\ ${ }^{1}$ Research and Development Centre, Vel Tech Dr. RR \& Dr. SR Technical University \\ Avadi, Chennai-600 062, Tamil Nadu, INDIA \\ sundarvtuegmail. com
}

\begin{abstract}
This paper derives new results for the global chaos synchronization of identical Sprott L systems (1994), identical Sprott M systems (1994) and non-identical Sprott L and M systems. Active control method has been deployed to achieve the global chaos synchronization of the identical and different Sprott $L$ and $M$ systems. Our synchronization results have been established using Lyapunov stability theory. Numerical plots have been presented to show the effectiveness of the active synchronization results derived in this paper for the Sprott L and M systems.
\end{abstract}

\section{KEYWORDS}

Chaos, Chaotic Systems, Synchronization, Active Control, Sprott-L system, Sprott-M system.

\section{INTRODUCTION}

Chaotic systems are nonlinear systems, which are characterized by the butterfly effect [1], viz. high sensitivity to small changes in the initial conditions of the systems. Chaos phenomenon has been extensively studied in the last two decades [1-23]. Chaos theory has been applied in various fields such as Computer Science, Biology, Microbiology, Ecology, Physics, Chemistry, Economics, Secure Communications, Image Processing and Robotics.

Synchronization of chaotic systems is a phenomenon that may occur when two or more chaotic oscillators are coupled or when a chaotic oscillator drives another chaotic oscillator.

In 1990, Pecora and Carroll [4] devised a novel scheme to synchronize two identical chaotic systems and showed that it was possible for some chaotic systems to be completely synchronized. From then on, chaos methods have been applied to various fields, viz. physical systems [5], chemical systems [6], ecological systems [7], secure communications [8-10], etc.

Since the seminal work by Pecora and Carroll [4], various methods have been proposed for the complete chaos synchronization such as OGY method [11], active control method [12-15], adaptive control method [16-20], backstepping method [21-22], sampled-data feedback synchronization method [23], time-delay feedback method [24], sliding mode control method [2528], etc. 
In this paper, new results have been derived for the complete synchronization for identical and different Sprott L and M chaotic systems using active nonlinear control. Using active control and Lyapunov stability theory, we achieve complete synchronization for identical Sprott L systems ([29], 1994), identical Sprott M systems ([29], 1994) and non-identical Sprott L and M systems.

\section{Problem Statement AND OUR Methodology}

Consider the master system described by the dynamics

$$
\dot{x}=A x+f(x)
$$

where $x \in R^{n}$ is the state of the system, $A$ is the $n \times n$ matrix of the system parameters and $f: R^{n} \rightarrow R^{n}$ is the nonlinear part of the system.

As the slave system, we consider the following chaotic system described by the dynamics

$$
\dot{y}=B y+g(y)+u
$$

where $y \in R^{n}$ is the state of the system, $B$ is the $n \times n$ matrix of the system parameters, $g: R^{n} \rightarrow R^{n}$ is the nonlinear part of the system and $u \in R^{n}$ is the active controller of the slave system.

If $A=B$ and $f=g$, then $x$ and $y$ are the states of two identical chaotic systems. If $A \neq B$ or $f \neq g$, then $x$ and $y$ are the states of two different chaotic systems.

In the active control method, we design a feedback controller $u$, which synchronizes the states of the master system (1) and the slave system (2) for all initial conditions $x(0), z(0) \in R^{n}$.

We define the complete synchronization error as

$$
e=y-x
$$

From (1), (2) and (3), we obtain the error dynamics as

$$
\dot{e}=B y-A x+g(y)-f(x)+u
$$

Thus, the complete synchronization problem is essentially to find a feedback controller $u$ so as to stabilize the error dynamics (4) for all initial conditions $e(0) \in R^{n}$.

Hence, we find a feedback controller $u$ so that

$$
\lim _{t \rightarrow \infty}\|e(t)\|=0 \text { for all } e(0) \in R^{n}
$$

We take as a candidate Lyapunov function

$$
V(e)=e^{T} P e,
$$


where $P$ is a positive definite matrix.

Note that $V: R^{n} \rightarrow R$ is a positive definite function by construction.

We suppose that the parameters of the master and slave system are known and that the states of both systems (1) and (2) can be measured.

We wish to find a feedback controller $u$ so that

$$
\dot{V}(e)=-e^{T} Q e,
$$

where $Q$ is a positive definite matrix. Then $\dot{V}: R^{n} \rightarrow R$ is a negative definite function.

Thus, by Lyapunov stability theory [30], the error dynamics (4) is globally exponentially stable.

Hence, it is immediate that the states of the master system (1) and the slave system (2) will be globally and exponentially synchronized.

\section{SYSTEMS DESCRIPTION}

In this section, we describe the chaotic systems studied in this paper, viz. Sprott $\mathrm{L}$ and $\mathrm{M}$ systems ([29], 1994).

The Sprott-L system is described by the 3D dynamics

$$
\begin{aligned}
& \dot{x}_{1}=x_{2}+a x_{3} \\
& \dot{x}_{2}=b x_{1}^{2}-x_{2} \\
& \dot{x}_{3}=c-x_{1}
\end{aligned}
$$

where $x_{1}, x_{2}, x_{3}$ are the states and $a, b, c$ are constant, positive parameters of the system.

The Sprott-L system (8) exhibits a strange chaotic attractor (see Figure 1), when the parameter values are taken as $a=3.9, b=0.9$ and $c=1$. 


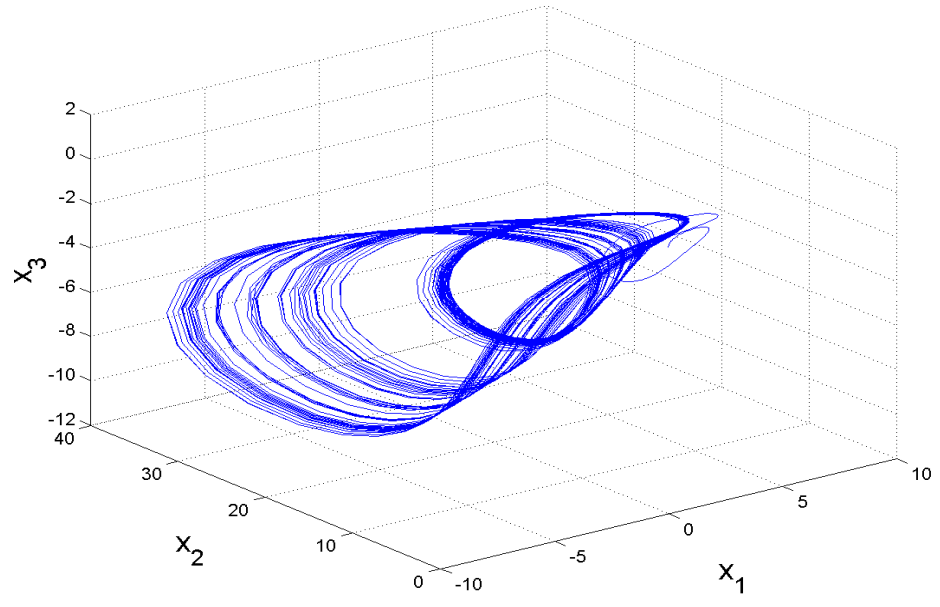

Figure 1. Strange Chaotic Attractor of the Sprott-L System

The Sprott-M system ([29], 1994) is described by the 3D dynamics

$$
\begin{aligned}
& \dot{x}_{1}=-x_{3} \\
& \dot{x}_{2}=-x_{1}^{2}-\alpha x_{2} \\
& \dot{x}_{3}=\beta+\gamma x_{1}+x_{2}
\end{aligned}
$$

where $x_{1}, x_{2}, x_{3}$ are the states and $\alpha, \beta, \gamma$ are constant, positive parameters of the system.

The Sprott-M dynamics (9) exhibits a chaotic attractor (see Figure 2), when the parameter values are taken as $\alpha=1, \beta=1.7$ and $\gamma=1.7$.

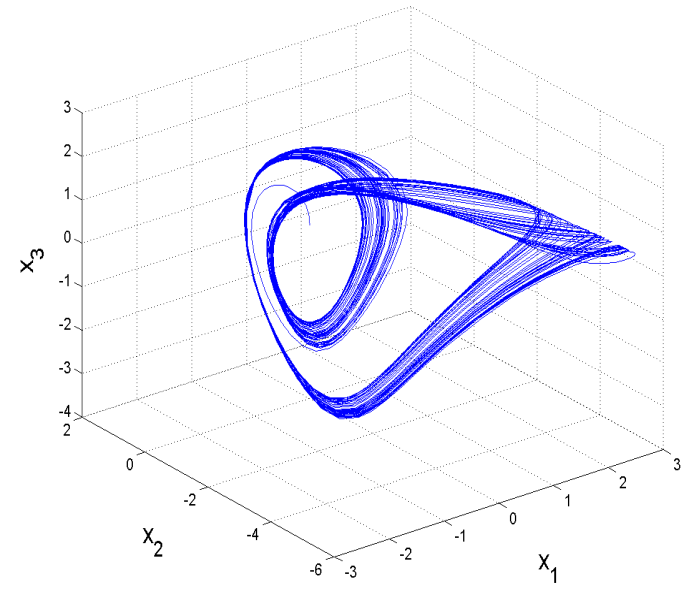

Figure 2. Strange Chaotic Attractor of the Sprott-M System 


\section{Global Chaos Synchronization of Identical Sprott-L Systems BY ACTIVE CONTROL}

\subsection{Theoretical Results}

In this section, we derive new results for the global chaos synchronization of two identical SprottL systems (1994) via the active control method.

Thus, the master system is described by the Sprott-L dynamics

$$
\begin{aligned}
& \dot{x}_{1}=x_{2}+a x_{3} \\
& \dot{x}_{2}=b x_{1}^{2}-x_{2} \\
& \dot{x}_{3}=c-x_{1}
\end{aligned}
$$

where $x_{1}, x_{2}, x_{3}$ are the state variables and $a, b, c$ are positive parameters of the system.

The slave system is described by the controlled Sprott-L dynamics

$$
\begin{aligned}
& \dot{y}_{1}=y_{2}+a y_{3}+u_{1} \\
& \dot{y}_{2}=b y_{1}^{2}-y_{2}+u_{2} \\
& \dot{y}_{3}=c-y_{1}+u_{3}
\end{aligned}
$$

where $y_{1}, y_{2}, y_{3}$ are the state variables and $u_{1}, u_{2}, u_{3}$ are the active controls to be designed.

The synchronization error $e$ is defined by

$$
\begin{aligned}
& e_{1}=y_{1}-x_{1} \\
& e_{2}=y_{2}-x_{2} \\
& e_{3}=y_{3}-x_{3}
\end{aligned}
$$

The error dynamics is obtained as

$$
\begin{aligned}
& \dot{e}_{1}=e_{2}+a e_{3}+u_{1} \\
& \dot{e}_{2}=-e_{2}+b\left(y_{1}^{2}-x_{1}^{2}\right)+u_{2} \\
& \dot{e}_{3}=-e_{1}+u_{3}
\end{aligned}
$$

We choose the active nonlinear controller as

$$
\begin{aligned}
& u_{1}=-e_{2}-a e_{3}-k_{1} e_{1} \\
& u_{2}=e_{2}-b\left(y_{1}^{2}-x_{1}^{2}\right)-k_{2} e_{2} \\
& u_{3}=e_{1}-k_{3} e_{3}
\end{aligned}
$$

where the gains $k_{i},(i=1,2,3)$ are positive constants. 
Substituting (14) into (13), the error dynamics simplifies to

$$
\begin{aligned}
& \dot{e}_{1}=-k_{1} e_{1} \\
& \dot{e}_{2}=-k_{2} e_{2} \\
& \dot{e}_{3}=-k_{3} e_{3}
\end{aligned}
$$

Next, we establish the following result.

Theorem4.1. The identical Sprott-L systems (10) and (11) are globally and exponentially synchronized for all initial conditions with the active controller defined by (12).

Proof. Consider the quadratic Lyapunov function defined by

$$
V(e)=\frac{1}{2} e^{T} e=\frac{1}{2}\left(e_{1}^{2}+e_{2}^{2}+e_{3}^{2}\right)
$$

which is a positive definite function on $R^{3}$.

Differentiating (16) along the trajectories of (15), we get

$$
\dot{V}(e)=-k_{1} e_{1}^{2}-k_{2} e_{2}^{2}-k_{3} e_{3}^{2}
$$

which is a negative definite function on $R^{3}$.

Thus, by Lyapunov stability theory [30], the error dynamics (17) is globally exponentially stable.

Hence, it follows that the identical Sprott-L systems (10) and (11) are globally and exponentially synchronized for all initial conditions with the active controller (12).

This completes the proof.

\subsection{Numerical Results}

For simulations, the fourth-order Runge-Kutta method with time-step $h=10^{-8}$ is deployed to solve the systems (10) and (11) with the active controller (14).

The feedback gains used in the equation (14) are chosen as

$$
k_{1}=5, k_{2}=5, k_{3}=5
$$

The parameters of the Sprott-L systems are chosen as

$$
a=3.9, \quad b=0.9, \quad c=1
$$

The initial conditions of the master system (10) are chosen as

$$
x_{1}(0)=1, \quad x_{2}(0)=-2, \quad x_{3}(0)=2
$$


The initial conditions of the slave system (11) are chosen as

$$
y_{1}(0)=3, \quad y_{2}(0)=6, \quad y_{3}(0)=-2
$$

Figure 3 shows the time-history of the synchronization errors $e_{1}, e_{2}, e_{3}$.

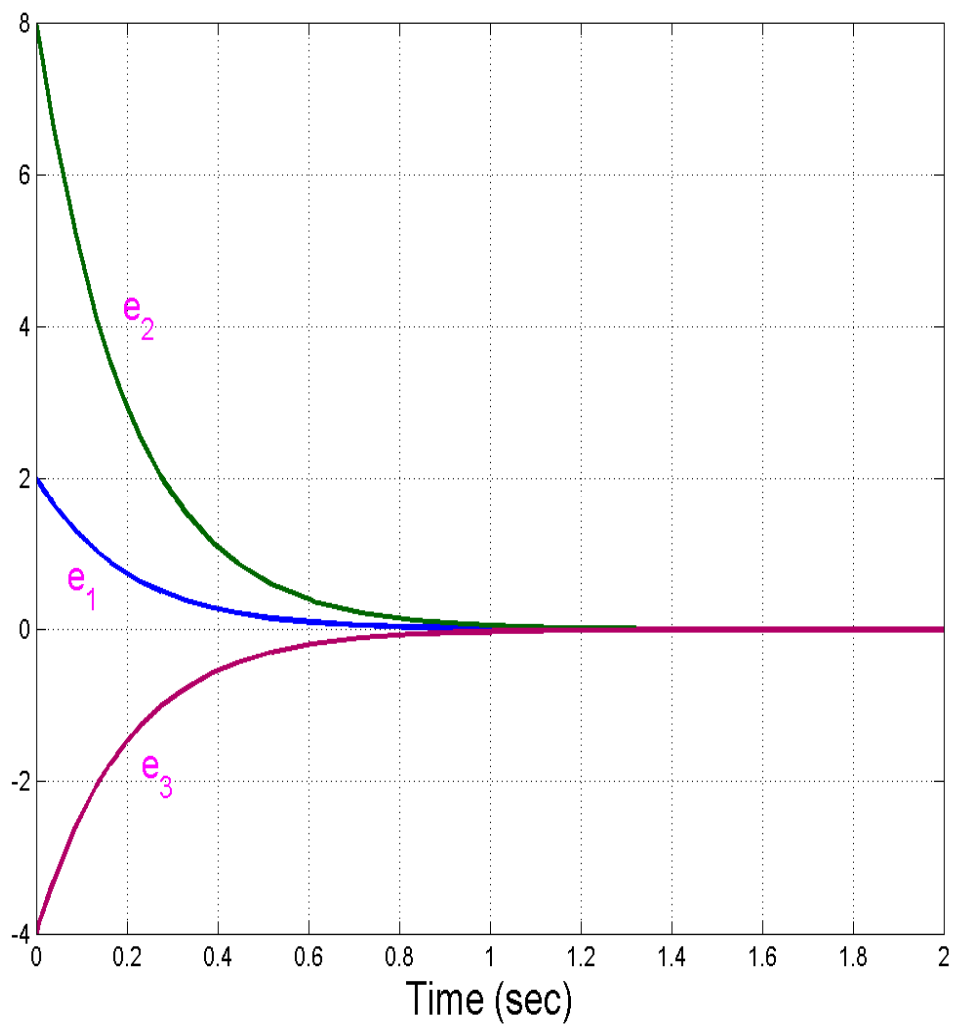

Figure 3. Time-History of the Synchronization Error $e_{1}, e_{2}, e_{3}$

\section{Global Chaos Synchronization of Identical SprotT-M Systems BY ACTIVE CONTROL}

\subsection{Theoretical Results}

In this section, we apply the active nonlinear control method for the synchronization of two identical Sprott-M systems (1994).

Thus, the master system is described by the Sprott-M dynamics 
International Journal of Control Theory and Computer Modelling (IJCTCM) Vol.2, No.4, July 2012

$$
\begin{aligned}
& \dot{x}_{1}=-x_{3} \\
& \dot{x}_{2}=-x_{1}^{2}-\alpha x_{2} \\
& \dot{x}_{3}=\beta+\gamma x_{1}+x_{2}
\end{aligned}
$$

where $x_{1}, x_{2}, x_{3}$ are the state variables and $\alpha, \beta, \gamma$ are positive parameters of the system. The slave system is described by the controlled Sprott-M dynamics

$$
\begin{aligned}
& \dot{y}_{1}=-y_{3}+u_{1} \\
& \dot{y}_{2}=-y_{1}^{2}-\alpha y_{2}+u_{2} \\
& \dot{y}_{3}=\beta+\gamma y_{1}+y_{2}+u_{3}
\end{aligned}
$$

where $y_{1}, y_{2}, y_{3}$ are the state variables and $u_{1}, u_{2}, u_{3}$ are the active controls to be designed.

The synchronization error $e$ is defined by

$$
\begin{aligned}
& e_{1}=y_{1}-x_{1} \\
& e_{2}=y_{2}-x_{2} \\
& e_{3}=y_{3}-x_{3}
\end{aligned}
$$

The error dynamics is obtained as

$$
\begin{aligned}
& \dot{e}_{1}=-e_{3}+u_{1} \\
& \dot{e}_{2}=-\alpha e_{2}-y_{1}^{2}+x_{1}^{2}+u_{2} \\
& \dot{e}_{3}=\gamma e_{1}+e_{2}+u_{3}
\end{aligned}
$$

We choose the active nonlinear controller as

$$
\begin{aligned}
& u_{1}=e_{3}-k_{1} e_{1} \\
& u_{2}=\alpha e_{2}+y_{1}^{2}-x_{1}^{2}-k_{2} e_{2} \\
& u_{3}=-\gamma e_{1}-e_{2}-k_{3} e_{3}
\end{aligned}
$$

where the gains $k_{i},(i=1,2,3)$ are positive constants.

Substituting (22) into (21), the error dynamics simplifies to

$$
\dot{e}_{i}=-k_{i} e_{i}, \quad(i=1,2,3)
$$

Theorem 5.1. The identical Sprott-M systems (18) and (19) are globally and exponentially synchronized for all initial conditions with the active nonlinear controller defined by (22).

Proof. Consider the quadratic Lyapunov function defined by

$$
V(e)=\frac{1}{2} e^{T} e=\frac{1}{2}\left(e_{1}^{2}+e_{2}^{2}+e_{3}^{2}\right)
$$


International Journal of Control Theory and Computer Modelling (IJCTCM) Vol.2, No.4, July 2012

which is a positive definite function on $R^{3}$.

Differentiating (24) along the trajectories of (23), we get

$$
\dot{V}(e)=-k_{1} e_{1}^{2}-k_{2} e_{2}^{2}-k_{3} e_{3}^{2}
$$

Clearly, $\dot{V}$ is a negative definite function on $R^{3}$.

Thus, by Lyapunov stability theory [30], the error dynamics (23) is globally exponentially stable.

This completes the proof.

\subsection{Numerical Results}

For simulations, the fourth-order Runge-Kutta method with time-step $h=10^{-8}$ is deployed to solve the systems (18) and (19) with the active nonlinear controller (22).

The feedback gains used in the equation (22) are chosen as $k_{i}=5,(i=1,2,3)$.

The parameters of the Sprott-M systems are chosen as

$$
\alpha=1, \beta=1.7, \gamma=1.7
$$

The initial conditions of the master system (20) are chosen as

$$
x_{1}(0)=2, \quad x_{2}(0)=4, \quad x_{3}(0)=-5
$$

The initial conditions of the slave system (21) are chosen as

$$
y_{1}(0)=6, \quad y_{2}(0)=-4, y_{3}(0)=11
$$

Figure 4 shows the time-history of the synchronization errors $e_{1}, e_{2}, e_{3}$. 
International Journal of Control Theory and Computer Modelling (IJCTCM) Vol.2, No.4, July 2012

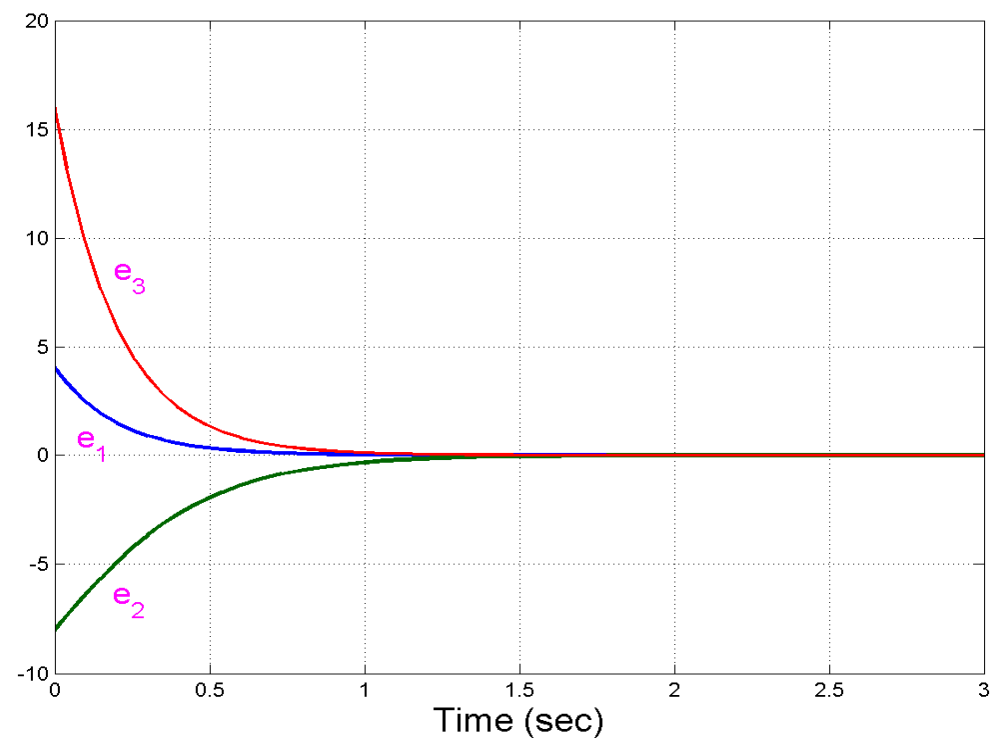

Figure 4. Time-History of the Synchronization Error $e_{1}, e_{2}, e_{3}$

\section{Global Chaos Synchronization of Sprott-L and Sprott-M SySTEMS BY ACTIVE CONTROL}

\subsection{Theoretical Results}

In this section, we apply the active nonlinear control method for the synchronization of the nonidentical Sprott-L and Sprott-M systems (1994).

Thus, the master system is described by the Sprott-L dynamics

$$
\begin{aligned}
& \dot{x}_{1}=x_{2}+a x_{3} \\
& \dot{x}_{2}=b x_{1}^{2}-x_{2} \\
& \dot{x}_{3}=c-x_{1}
\end{aligned}
$$

where $x_{1}, x_{2}, x_{3}$ are the state variables and $a, b, c$ are positive parameters of the system.

The slave system is described by the controlled Sprott-M dynamics

$$
\begin{aligned}
& \dot{y}_{1}=-y_{3}+u_{1} \\
& \dot{y}_{2}=-y_{1}^{2}-\alpha y_{2}+u_{2} \\
& \dot{y}_{3}=\beta+\gamma y_{1}+y_{2}+u_{3}
\end{aligned}
$$

where $y_{1}, y_{2}, y_{3}$ are the state variables, $\alpha, \beta, \gamma$ are positive parameters and $u_{1}, u_{2}, u_{3}$ are the active nonlinear controls to be designed. 
The synchronization error $e$ is defined by

$$
\begin{aligned}
& e_{1}=y_{1}-x_{1} \\
& e_{2}=y_{2}-x_{2} \\
& e_{3}=y_{3}-x_{3}
\end{aligned}
$$

The error dynamics is obtained as

$$
\begin{aligned}
& \dot{e}_{1}=-y_{3}-x_{2}-a x_{3}+u_{1} \\
& \dot{e}_{2}=-y_{1}^{2}-b x_{1}^{2}-\alpha y_{2}+x_{2}+u_{2} \\
& \dot{e}_{3}=\beta-c+\gamma y_{1}+x_{1}+y_{2}+u_{3}
\end{aligned}
$$

We choose the active nonlinear controller as

$$
\begin{aligned}
& u_{1}=y_{3}+x_{2}+a x_{3}-k_{1} e_{1} \\
& u_{2}=y_{1}^{2}+b x_{1}^{2}+\alpha y_{2}-x_{2}-k_{2} e_{2} \\
& u_{3}=-\beta+c-\gamma y_{1}-x_{1}-y_{2}-k_{3} e_{3}
\end{aligned}
$$

where the gains $k_{i},(i=1,2,3)$ are positive constants.

Substituting (32) into (31), the error dynamics simplifies to

$$
\begin{aligned}
& \dot{e}_{1}=-k_{1} e_{1} \\
& \dot{e}_{2}=-k_{2} e_{2} \\
& \dot{e}_{3}=-k_{3} e_{3}
\end{aligned}
$$

Theorem 6.1. The Sprott-L system (26) and Sprott-M system (27) are globally and exponentially synchronized for all initial conditions with the active controller defined by (30).

Proof. We consider the quadratic Lyapunov function defined by

$$
V(e)=\frac{1}{2} e^{T} e=\frac{1}{2}\left(e_{1}^{2}+e_{2}^{2}+e_{3}^{2}\right)
$$

which is a positive definite function on $R^{3}$.

Differentiating (32) along the trajectories of (31), we get

$$
\dot{V}(e)=-k_{1} e_{1}^{2}-k_{2} e_{2}^{2}-k_{3} e_{3}^{2}
$$

which is a negative definite function on $R^{3}$.

Thus, by Lyapunov stability theory [30], the error dynamics (33) is globally exponentially stable.

This completes the proof. 
International Journal of Control Theory and Computer Modelling (IJCTCM) Vol.2, No.4, July 2012

\subsection{Numerical Results}

For simulations, the fourth-order Runge-Kutta method with time-step $h=10^{-8}$ is deployed to solve the systems (26) and (27) with the active nonlinear controller (30).

The feedback gains used in the equation (32) are chosen as $k_{i}=5,(i=1,2,3)$.

The parameters of the Sprott-L systems are chosen as

$$
a=3.9, \quad b=0.9, \quad c=1
$$

The parameters of the Sprott-M systems are chosen as

$$
\alpha=1, \beta=1.7, \gamma=1.7
$$

The initial conditions of the master system (26) are chosen as

$$
x_{1}(0)=2, \quad x_{2}(0)=4, \quad x_{3}(0)=-1
$$

The initial conditions of the slave system (27) are chosen as

$$
y_{1}(0)=-2, \quad y_{2}(0)=7, y_{3}(0)=6
$$




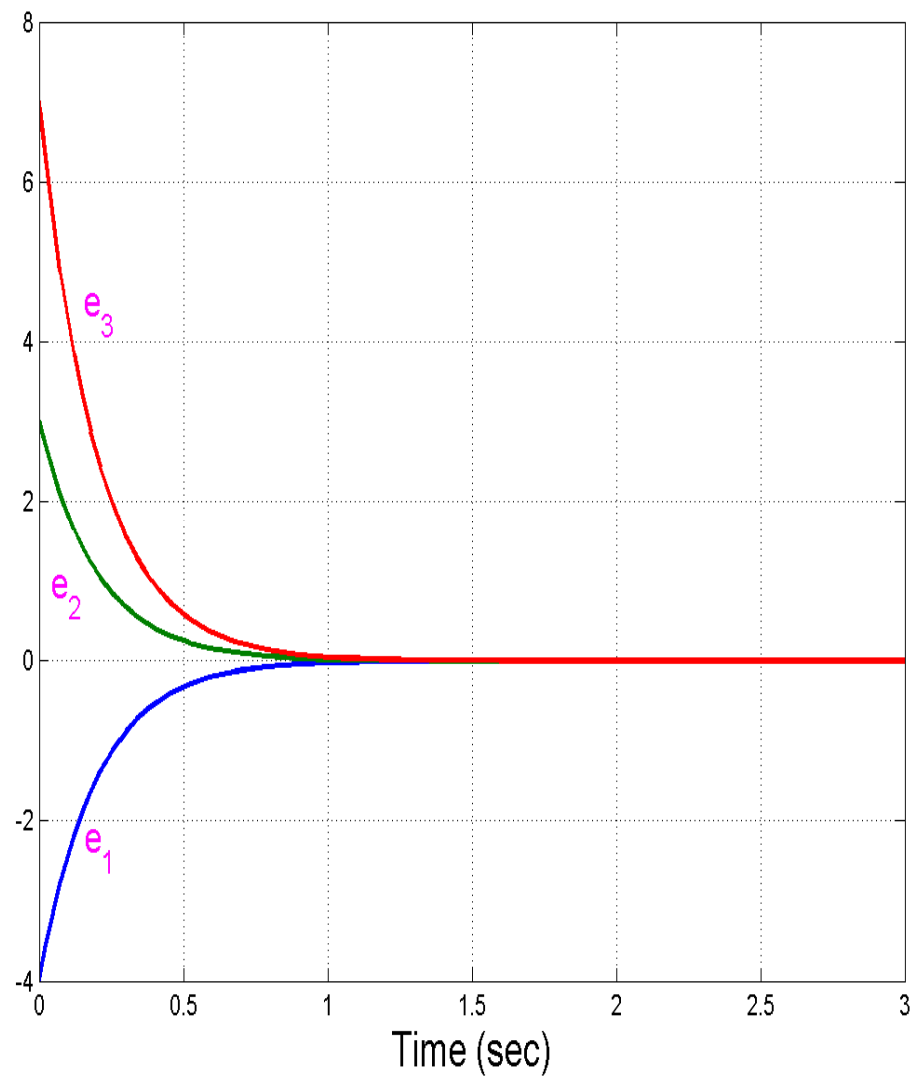

Figure 5. Time-History of the Synchronization Error $e_{1}, e_{2}, e_{3}$

Figure 5 shows the time-history of the synchronization errors $e_{1}, e_{2}, e_{3}$.

\section{Conclusions}

In this paper, we derived new results for the global chaos synchronization for the Sprott-L and Sprott-M systems using active control method and we established the synchronization results with the help of Lyapunov stability theory. Numerical simulations have been shown to illustrate the effectiveness of the complete synchronization schemes derived in this paper for the Sprott-L and Sprott-M systems.

\section{REFERENCES}

[1] Alligood, K.T., Sauer, T. \& Yorke, J.A. (1997) Chaos: An Introduction to Dynamical Systems, Springer, New York.

[2] Rössler, O.E. (1979) “An equation for hyperchaos,” Physics Letters A, Vol. 71, pp 155-157.

[3] Buscarino, A., Fortuna, L. \& Frasca, M. (2009) "Experimental robust synchronization of hyperchaotic circuits," Physica D: Nonlinear Phenomena, Vol. 238, No. 18, pp 1917-1922.

[4] Pecora, L.M. \& Carroll, T.L. (1990) "Synchronization in chaotic systems", Phys. Rev. Lett., Vol. 64, pp 821-824. 
International Journal of Control Theory and Computer Modelling (IJCTCM) Vol.2, No.4, July 2012

[5] Lakshmanan, M. \& Murali, K. (1996) Nonlinear Oscillators: Controlling and Synchronization, World Scientific, Singapore.

[6] Han, S.K., Kerrer, C. \& Kuramoto, Y. (1995) "Dephasing and burstling in coupled neural oscillators", Phys. Rev. Lett., Vol. 75, pp 3190-3193.

[7] Blasius, B., Huppert, A. \& Stone, L. (1999) "Complex dynamics and phase synchronization in spatially extended ecological system”, Nature, Vol. 399, pp 354-359.

[8] Feki, M. (2003) "An adaptive chaos synchronization scheme applied to secure communication", Chaos, Solitons and Fractals, Vol. 18, pp 141-148.

[9] Murali, K. \& Lakshmanan, M. (1998) "Secure communication using a compound signal from generalized synchronizable chaotic systems", Phys. Rev. Lett. A, Vol. 241, pp 303-310.

[10] Yang, T. (2004) "A survey of chaotic secure communication systems," International Journal of Computational Cognition, Vol. 2, No. 2, pp 81-130.

[11] Ott, E., Grebogi, C. \& Yorke, J.A. (1990) “Controlling chaos”, Phys. Rev. Lett., Vol. 64, pp 11961199.

[12] Ho, M.C. \& Hung, Y.C. (2002) "Synchronization of two different chaotic systems by using generalized active control", Physics Letters A, Vol. 301, pp 424-428.

[13] Chen, H.K. (2005) "Global chaos synchronization of new chaotic systems via nonlinear control", Chaos, Solitons \& Fractals, Vol. 23, pp 1245-1251.

[14] Sundarapandian, V. (2011) "Global chaos synchronization of four-scroll and four-wing chaotic attractors by active nonlinear control," International Journal on Computer Science and Engineering, Vol. 3, No. 5, pp. 2145-2155.

[15] Sundarapandian, V. (2011) "Global chaos synchronization of Li and Liu-Chen-Liu chaotic systems by active nonlinear control," International Journal of Advances in Science and Technology, Vol. 3, No. 1, pp. 1-12.

[16] Liao, T.L. \& Tsai, S.H. (2000) "Adaptive synchronization of chaotic systems and its applications to secure communications", Chaos, Solitons and Fractals, Vol. 11, pp 1387-1396.

[17] Sundarapandian, V. (2011) "Adaptive control and synchronization of hyperchaotic Cai system", International Journal of Control Theory and Computer Modelling, Vol. 1, No. 1, pp 1-13.

[18] Sundarapandian, V. (2011) "Adaptive synchronization of hyperchaotic Lorenz and hyperchaotic Liu systems”, International Journal of Instrumentation and Control Systems, Vol. 1, No. 1, pp 1-18.

[19] Sundarapandian, V. (2011) "Adaptive control and synchronization of Liu's four-wing chaotic system with cubic nonlinearity," International Journal of Computer Science, Engineering and Applications, Vol. 1, No. 4, pp 127-138.

[20] Sundarapandian, V. \& Karthikeyan, R. (2011) "Global chaos synchronization of Pan and Lü chaotic systems via adaptive control," International Journal of Information Technology, Convergence and Services, Vol. 1, No. 5, pp. 49-66.

[21] Yu, Y.G. \& Zhang, S.C. (2006) "Adaptive backstepping synchronization of uncertain chaotic systems", Chaos, Solitons and Fractals, Vol. 27, pp 1369-1375.

[22] Wu, X. \& Lü, J. (2003), "Parameter identification and backstepping control of uncertain Lü system," Chaos, Solitons and Fractals, Vol. 18, pp 721-729.

[23] Yang, T. \& Chua, L.O. (1999) "Control of chaos using sampled-data feedback control", Internat. J. Bifurcat. Chaos, Vol. 9, pp 215-219.

[24] Park, J.H. \& Kwon, O.M. (2003) "A novel criterion for delayed feedback control of time-delay chaotic systems", Chaos, Solitons and Fractals, Vol. 17, pp 709-716.

[25] Sundarapandian, V. (2011) "Global chaos synchronization of four-wing chaotic systems by sliding mode control", International Journal of Control Theory and Computer Modelling, Vol. 1, No. 1, pp 15-31.

[26] Sundarapandian, V. (2011) "Global chaos synchronization of Pehlivan systems by sliding mode control”, International Journal on Computer Science and Engineering, Vol. 3, No. 5, pp 2163-2169.

[27] Sundarapandian, V. (2011) "Sliding mode controller design for the synchronization of ShimizuMorioka chaotic systems", International Journal of Information Sciences and Techniques, Vol. 1, No. 1, pp 20-29.

[28] Sundarapandian, V. \& Sivaperumal, S. (2012) "Anti-synchronization of four-wing chaotic systems via sliding mode control", International Journal of Automation and Computing, Vol. 9, No. 3, pp 274-279.

[29] Sprott, J.C. (1994) “Some simple chaotic flows”, Phy. Review E, Vol. 50, No. 2, pp 647-650.

[30] Hahn, W. (1967) The Stability of Motion, Springer, New York. 
International Journal of Control Theory and Computer Modelling (IJCTCM) Vol.2, No.4, July 2012

\section{Author}

Dr. V. Sundarapandian earned his Doctor of Science degree in Electrical and Systems Engineering from Washington University, St. Louis, USA in May 1996. He is a Professor at the R \& D Centre at Vel Tech Dr. RR \& Dr. SR Technical University, Chennai, Tamil $\mathrm{Nadu}$, India. He has published over 270 papers in refereed international journals. He has published over 170 papers in National and International Conferences. He is the Editor-inChief of the AIRCC Journals - International Journal of Instrumentation and Control Systems, International Journal of Control Systems and Computer Modelling, and International Journal of Information Technology, Control and Automation. His research interests are Linear and Nonlinear Control Systems, Chaos Theory and Control, Soft Computing, Optimal Control, Operations Research, Mathematical Modelling and Scientific Computing. He has delivered several Keynote lectures on nonlinear control systems, chaos theory, mathematical modelling and scientific computing using MALTAB/SCILAB 